\title{
Elaborate study design leads to improved conclusion
}

\author{
Sae-Cheol Oh \\ Department of Anesthesiology and Pain Medicine, Daejeon St. Mary's Hospital, College of Medicine, The Catholic \\ University of Korea, Daejeon, Korea
}

Taking care of surgical patients with temporary mental dysfunction is one of the biggest challenges for the anesthesiologist in the recovery room. This temporary mental dysfunction presents with two different faces: one is emergence agitation (EA) in pediatric patients and the other is delirium in geriatric patients.

EA is the clinical condition of pediatric patients who are suffering from temporary mental dysfunction after general anesthesia. It is a transient dysfunction of the brain. However, it is essential to differentiate EA from the similar condition of agitation due to insufficient pain control in the recovery room. The clinical presentations of EA and agitation due to pain are so similar in presentation that differentiation of the two conditions is very difficult in pediatric patients.

Furthermore, the sedation caused by anesthetic drugs is another contributing factor to the onset and degree of EA. Sedation resulting from anesthetic drugs can mask or delay the onset of EA.

If we wish to study the effect of a test drug on EA in pediatric patients in the recovery room, it is necessary to prove that there are no differences in the degree of agitation resulting from insufficient pain control and sedation from residual anesthetics between the control and experimental group. If this is not done, it would be very difficult to interpret the results in relation to EA in the experimental group. Particularly, if the test drug has significant greater sedative effect compared to the control drug,

Corresponding author: Sae-Cheol Oh, M.D., Ph.D.

Department of Anesthesiology and Pain Medicine, Daejeon St. Mary's Hospital, College of Medicine, The Catholic University of Korea, 64, Daeheung-ro, Jung-gu, Daejeon 34943, Korea

Tel: 82-42-220-9400, Fax: 82-42-220-3400

E-mail: oscane0822@gmail.com

Received: November 4, 2015.

Revised: March 21, 2016 (1st); April 26, 2016 (2nd).

Accepted: April 26, 2016.

Korean J Anesthesiol 2016 October 69(5): 535

http://dx.doi.org/10.4097/kjae.2016.69.5.535 it would be impossible to calculate how much the test drug had contributed to the onset and degree of EA, reflected in the EA scores, in the children.

In the case of agitation due to incomplete pain control, regional anesthesia or use of the same amount of pain-killer would provide proof of the same degree of pain in the pediatric patients in the recovery room. In addition, the sedation score would be a better parameter to use to demonstrate the same degree of sedation in the two groups.

A recent paper studied EA in pediatric patients in the recovery room. The title of the article is "A comparison of postoperative emergence agitation between sevoflurane and thiopental anesthesia induction in pediatric patients" (Korean J Anesthesiol 2015 Aug; 68(4): 373-378).

In terms of the two factors mentioned above, some questions arise regarding the results of this paper. Although the author discussed his rationale for using the different EA scales, a fourpoint agitation scale at 5 minutes and the Pediatric Anesthesia Emergence Delirium scale at 20 minutes, it would be impossible for the author to rule out the possibility of overestimation of the number of children receiving low scores for EA in the thiopental group. That is to say, the sedation from the residual effect of the thiopental was regarded as no EA at 5 minutes in the experimental group in the recovery room. If sedation scores were measured in both groups, the effect of sedation would be ruled out from the EA. Additionally, the results would be improved if the data on the pain-killer used in the recovery room was mentioned in the paper.

In conclusion, an elaborate study design to rule out the effects of sedation and pain on EA in the recovery room would assist in the generation of improved results in the paper.

(c) This is an open-access article distributed under the terms of the Creative Commons Attribution Non-Commercial License (http://creativecommons.org/ licenses/by-nc/4.0/), which permits unrestricted non-commercial use, distribution, and reproduction in any medium, provided the original work is properly cited. 